\section{Regards sur l'économie allemande}

Bulletin économique du CIRAC

$65 \mid 2004$

Varia

\title{
2003 : des réformes, mais pas encore la fin de l'immobilisme
}

\section{Stefanie Wahl}

Traducteur : Isabelle Bourgeois

\section{OpenEdition \\ Journals}

Édition électronique

URL : http://journals.openedition.org/rea/3937

DOI : 10.4000/rea.3937

ISBN : 978-2-8218-0826-3

ISSN : 1965-0787

Éditeur

CIRAC

Édition imprimée

Date de publication : 1 mars 2004

Pagination : $5-10$

ISSN : 1156-8992

\section{Référence électronique}

Stefanie Wahl, « 2003 : des réformes, mais pas encore la fin de l'immobilisme », Regards sur l'économie allemande [En ligne], 65 I mars 2004, mis en ligne le 29 octobre 2009, consulté le 02 mai 2019. URL http://journals.openedition.org/rea/3937 ; DOI : 10.4000/rea.3937 


\title{
2003 : des réformes, mais pas encore la fin de l'immobilisme
}

\author{
Stefanie Wahl
}

\begin{abstract}
L'Allemagne bouge... du moins un peu. Après des décennies d'immobilisme, les responsables politiques ont mis en route l'an passé une série d'importantes réformes de politique économique, sociale, fiscale et de l'emploi, s'attaquant enfin à cette rénovation structurelle si longtemps repoussée. II est vrai qu'il y avait urgence. Car depuis les années 70 , le contexte économique, la démographie et la société avaient subi de profondes mutations, mais l'opinion et la sphère politique avait préféré les ignorer, voire les refouler. Les tendances étaient pourtant nettes : la population allemande vieillissait rapidement, captant un volume croissant de transferts publics; sur le marché mondial, la concurrence s'avivait autour de ces facteurs de production rares que sont le capital et le savoir ; avec le progrès des technologies de l'information et de la communication, l'automatisation commençait à se substituer au travail humain. Mais les Allemands avaient préféré nier l'évidence pour se crisper sur leurs institutions et structures établies. Le résultat ne s'est pas fait attendre: un chômage en hausse sensible, une politique de l'emploi inefficace, des systèmes de protection sociale à la dérive, des prélèvements fiscaux et sociaux de plus en plus lourds, un déficit public qui ne cesse de se creuser, un système de formation initiale et professionnelle de moins en moins performant, et une dynamique de croissance ralentie.
\end{abstract}

Au plus tard depuis le début de la législature 2002, aussi bien les responsables politiques que la majorité de l'opinion avaient compris que la situation ne pouvait plus continuer ainsi. Si l'Allemagne voulait préserver durablement son niveau de prospérité et de confort social, il lui fallait adapter ses structures à l'évolution des réalités. Tous avaient compris également que cela ne pouvait se faire qu'au prix de douloureux sacrifices.

Or les politiques, sachant qu'ils risquaient de perdre leur crédibilité dans l'opinion s'ils imposaient brusquement à la population des réformes forcément radicales après tant d'années d'immobilisme, ont délégué cette mission ingrate à des commissions d'experts indépendants. La réforme du marché du travail avait ainsi été confiée à la "Commission Hartz », présidée par Peter Hartz, membre du directoire de Volkswagen. Pour la réforme des systèmes de protection sociale, le gouvernement fédéral avait instauré la " Commission Rürup », dirigée par le social-démocrate Bert Rürup, professeur d'économie sociale et membre du Conseil des Sages ; l'opposition créait de son côté la "Commission Herzog " sous la houlette de l'ancien président de la République, Roman Herzog, qui fut également juge du Tribunal constitutionnel fédéral.

Ces commissions ont toutes trois élaboré des propositions de réformes conséquentes, argumentées et souvent innovantes, qui contribuent à alimenter durablement le débat allemand sur les réformes structurelles. Alors que les divers projets de réforme de la protection sociale sont encore soit en débat parlementaire, soit en cours de discussion, les propositions de la Commission Hartz pour le marché de l'emploi ont d'ores et déjà été adoptées avec quelques modifications par le Bundestag et le Bundesrat.
Stefanie Wahl

est chargée de recherche à l'Institut für Wirtschaft und Gesellschaft, Bonn. Elle fut membre de la commission „Nouvelle économie sociale de marché" qui avait été instituée auprès du directoire du parti CDU entre janvier et septembre 2001.

Trois commissions pour élaborer des réformes

Seules les propositions Hartz ont abouti 


\section{Emploi : la moitié du chemin}

Incitations drastiques au retour en emploi

Restructuration de l'Office de Nuremberg

Reste à traiter les causes structurelles du chômage

C'est la tâche des partenaires sociaux

Vers un segment à bas salaires
Les « réformes Hartz » n'ont pas encore produit leurs effets dans les statistiques ; mais à moyen terme, elles devraient modifier sensiblement le marché de l'emploi. La réduction de la durée de versement des allocations chômage, la fusion de l'aide au chômeur (Arbeitslosenhilfe) et de l'aide sociale (Sozialhilfe) en une seule catégorie de revenus de substitution (Arbeitslosengeld II) d'un faible montant, l'obligation faite au bénéficiaire de ce revenu d'accepter toute proposition d'emploi, même à un niveau de rémunération inférieur au salaire conventionnel de branche - toutes ces mesures retirent leur fondement à la propension qu'avaient de nombreux chômeurs à épuiser leurs droits à un revenu de substitution et à ne se lancer que mollement dans la recherche d'un emploi.

Parallèlement, l'Office fédéral du Travail (Bundesanstalt für Arbeit, Nuremberg), jusqu'ici une administration gestionnaire de l'assurance chômage comme des mesures en faveur de l'emploi, sera transformé en un prestataire de services de placement des ressources humaines et rebaptisé Agence fédérale pour l'emploi (Bundesagentur für Arbeit). La transformation des antennes régionales de I'ANPE allemande en Job Center et la création d'agences d'intérim publiques (Personal-Service-Agenturen,) ont pour objectif d'accélérer le placement des chômeurs et d'en accroître l'efficience.

Ajoutées à cette restructuration, l'institution des Ich-AG, la simplification du régime des Mini-Jobs au sens strict comme au sens large ou la libéralisation de certaines activités artisanales qui ne sont plus soumises, désormais, aux règles corporatistes d'un Code de l'artisanat millénaire, posent les jalons d'un futur segment à bas salaires. Enfin, l'assouplissement de la protection contre le licenciement contribue à son tour à la dérégulation du marché du travail.

Néanmoins, l'espoir formulé initialement par la Commission Hartz - remettre en emploi deux millions de chômeurs en trois ans grâce aux mesures proposées restera lettre morte. En effet, nombreuses sont les propositions de la Commission à avoir été vidées de leur substance au cours des débats parlementaires. Plus important encore : conformément aux missions de la Commission Hartz, les lois qui sont issues de ses travaux ne peuvent remédier qu'à une partie des problèmes du marché allemand de l'emploi.

L'essentiel reste à faire : traiter les causes mêmes du chômage, comme le trop faible écart salarial, la dérive des coûts salariaux annexes ou l'inadéquation des qualifications. Enfin, le marché du travail allemand est l'un des plus réglementés du monde. La responsabilité en revient à un système de négociation collective dont le pivot est le principe constitutionnel de l'autonomie des partenaires sociaux en matière de macro-régulation sociale ; il ne peut donc être adapté aux mutations du travail sans l'assentiment exprès des partenaires sociaux. L'avenir du marché du travail allemand se trouve aussi entre leurs mains. C'est à eux qu'incombe désormais la responsabilité de sa dérégulation.

\section{Santé : la réforme structurelle reste à faire}

La Loi de modernisation de l'assurance maladie légale...
Les membres de la Commission Rürup n'ayant pas réussi à s'accorder sur une réforme du système de protection maladie, une nouvelle hausse des cotisations devenait nécessaire en 2004 pour contenir le déficit de l'assurance maladie légale. Mais elle aurait mis en danger les effets bénéfiques escomptés par les réformes Hartz. Pour néanmoins remédier dans l'immédiat à la dérive des coûts de santé, Bundestag et Bundesrat ont adopté à l'automne 2003 une Loi de modernisation de l'assurance maladie obligatoire (Gesetz zur Modernisierung der gesetzlichen Krankenversicherung). Son objectif étant de garantir les recettes des caisses tout en réduisant le niveau des cotisations, elle accroît la participation financière des assurés en introduisant une taxe dite de cabinet médical 
(Praxisgebühr), en haussant une série de tickets modérateurs et en supprimant la prise en charge de certaines prestations. Elle innove même en incitant les assurés à adopter un comportement responsable grâce à un système de bonus et de franchise.

Mais aucune de ces mesures n'aborde la réforme de fond de la structuration des recettes et des dépenses. On peut donc se demander à juste titre si l'objectif d'une compression durable du niveau des cotisations pourra réellement être atteint, d'autant plus que la mise en œuvre des nouvelles mesures accroît les coûts d'administration et de gestion pour les caisses. De nouvelles réformes, structurelles, seront donc indispensables, à l'instar des propositions émanant des Commissions Rürup, Herzog ou du parti CDU.

Au cœur des projets de réforme structurelle du financement de la protection maladie, deux modèles sont actuellement en débat : la prime forfait santé (pauschale Gesundheitsprämie) et l'assurance citoyenne (Bürgerversicherung). La prime forfait santé prévoit un montant mensuel de cotisation identique pour tous les assurés. Quant à la part de la cotisation maladie à charge de l'employeur, elle sera reversée par celui-ci à l'assuré ; et le total des cotisations sera soumis à impôt. Les assurés à faibles revenus se verraient verser une prime compensatoire financée par l'impôt. Sur le financement de cette prime forfaitaire, les avis divergent : la Commission Rürup préconise un système de répartition classique, le parti CDU y introduirait des éléments de capitalisation; quant à la Commission Herzog, elle plaide pour la seule capitalisation à terme.

A l'opposé, l'assurance citoyenne que propose une partie de la Commission Rürup soumettrait à cotisation tous les revenus, quelle que soit leur nature. Et elle serait obligatoire pour tous. Autrement dit: les professions libérales et les fonctionnaires, affiliés aux caisses privées, devraient cotiser eux aussi au régime légal de l'assurance maladie. Ce dernier serait alors généralisé, et le régime privé, supprimé.

En ne faisant plus supporter le financement de l'assurance maladie par les seuls salaires, c'est-à-dire en déconnectant les coûts de santé des coûts salariaux, les diverses versions du modèle de la prime forfait santé sont nettement plus favorables à l'emploi que l'assurance citoyenne. Car bien qu'elle diversifie l'assiette des cotisations, cette dernière n'en reste pas moins préjudiciable à l'emploi, puisque la hausse des coûts de santé ou la baisse du nombre d'actifs occupés accroît d'autant le poids des coûts salariaux annexes. Mais il y a plus problématique encore : aucun des projets de réforme en débat ne contribue réellement à renforcer l'efficience de la protection maladie, à en comprimer le coût ni à promouvoir un comportement responsable de la part de l'assuré ou du système de soins. Autrement dit : l'essentiel reste à faire.

\section{Retraites : encore trop de répartition et trop peu de capitalisation}

Deux ans seulement après la dernière réforme des retraites qui avait introduit des éléments de capitalisation dans le système de répartition (" retraite Riester »), la menace d'une hausse des cotisations en 2004 appelle des mesures d'urgence. En décembre 2003, Bundestag et Bundesrat adoptent donc un " programme d'urgence " visant à combler le trou des caisses de retraite et à stabiliser le niveau des cotisations. II impose aux retraités d'importants sacrifices : la hausse annuelle du montant des retraites est retardée de juillet 2004 à janvier 2005, le versement des pensions aux nouveaux retraités est repoussé du début à la fin du mois, et tous sont soumis au taux maximum de cotisation au régime d'assurance dépendance légal.

Comme ces mesures ne garantissent pas la stabilisation à terme des dépenses du régime légal des retraites, le gouvernement fédéral a soumis au parlement en décembre 2003 un projet de Loi sur la soutenabilité de l'assurance retraite
... élude la question-clef : l'avenir de son financement

En débat : prime forfait santé...

... ou assurance citoyenne?

Aucun

des deux modèles ne suffit

Un programme d'urgence pour les retraites en 2004 ...

... et un projet de loi sur la soutenabilité des retraites 
La question de l'âge légal de la retraite, repoussée à 2008

En projet : harmonisation fiscale entre revenus et retraites

Le système par répartition ne peut être maintenu en l'état

Vers une simplification radicale du code fiscal allemand?

Baisse anticipée des impôts en 2004

(Rentenversicherungsnachhaltigkeitsgesetz), actuellement en débat. Ce texte s'inspire de certaines propositions formulées par les Commissions Rürup et Herzog. Le pivot en est ce « facteur de soutenabilité à déterminante démographique » (demographiebestimmter Nachhaltigkeitsfaktor) qui vise à abaisser le niveau des retraites et à comprimer la hausse du taux de cotisation. Concrètement, cela signifie que le mode de calcul des retraites intègrera dorénavant un nouveau facteur: la relation entre le nombre de cotisants et le nombre de retraités.

Le projet de loi prévoit en outre que les chômeurs ou les bénéficiaires des mesures de temps partiel pour salariés âgés (Altersteilzeit) ne pourront faire valoir leurs droits au départ à la retraite anticipée qu'à 63 ans au lieu de 60 actuellement ; de même, les années de formation ne seront plus prises en compte dans le calcul des annuités. Par contre, ce projet n'a pas retenu la hausse progressive de l'âge légal de départ à la retraite - de 65 ans actuellement à 67 ans en 2030 - que préconisaient les deux commissions. Toutefois, le gouvernement fédéral s'est engagé à remettre cette question à l'ordre du jour en 2008.

Depuis décembre 2003 également, un autre projet de loi se trouve en débat parlementaire : la Loi sur les revenus des personnes âgées (Alterseinkünftegesetz), qui vise à simplifier la mise en œuvre de la " retraite Riester » et à défiscaliser progressivement les cotisations versées au titre de l'assurance retraite. En contrepartie, le taux d'imposition des pensions de retraite sera progressivement augmenté, celles-ci devant à terme être soumises au régime fiscal général des revenus. Mais il ne s'agit pour l'instant que d'un projet de loi.

Toutes les parties impliquées dans ces réformes préconisent le maintien d'un système légal d'assurance retraite par répartition et assis sur les salaires. Mais cela ne permettra ni de réduire les tensions sur le marché de l'emploi ni encore moins de créer un environnement économique propice à la constitution de ce capital pourtant indispensable à une population vieillissante et en diminution constante. Par ailleurs, le maintien d'un système financé majoritairement par la répartition se soldera par une hausse des taux de cotisation dont pâtiront invariablement les générations futures : elles n'auront plus alors la marge de manœuvre qui leur permettrait de constituer le capital-retraite nécessaire pour s'assurer un certain niveau de vie. Seule une réforme de fond permettra donc de garantir durablement l'avenir des retraites. Elle se fait attendre.

\section{Fiscalité : petite baisse en 2004 et grands projets futurs}

Pour relancer la croissance, Bundestag et Bundesrat ont décidé en décembre 2003 d'avancer partiellement d'un an le troisième et dernier volet de la loi de réforme fiscale adoptée en juillet 2000. Dès 2004, le taux minimum d'imposition est donc passé de $19,9 \%$ à $16,0 \%$, le taux plafond étant ramené de $48,5 \%$ à $45,0 \%$. En 2005 , ces taux passeront à respectivement $15,0 \%$ et $42,0 \%$. La baisse des impôts est contre-financée par une réduction des subventions, des privatisations et le recours à la dette.

Malgré l'anticipation de la réduction des prélèvements fiscaux, le taux marginal d'imposition des particuliers et, surtout, des entreprises, reste élevé en comparaison internationale. A ce problème s'ajoute l'opacité du système fiscal. Experts et hommes politiques de l'opposition ont donc formulé diverses propositions pour réformer en profondeur le code fiscal allemand. Tous plaident pour une réduction des taux d'imposition et une simplification radicale de la fiscalité. De tous ces projets, c'est celui de l'ancien juge au Tribunal constitutionnel fédéral, Paul Kirchhoff, qui va le plus loin : il prône non seulement un taux unique d'imposition pour les particuliers et les entreprises, mais propose aussi de ramener la classification des revenus à une seule catégorie. Quelques fiscalistes chrétiens-démocrates et libéraux, pour leur part, plaident pour une tarification à trois niveaux et une harmonisation plus poussée entre impôts sur le revenu et 
impôt sur les sociétés. Quant au parti CSU, il favorise le maintien de l'actuel système d'imposition linéaire et progressif. Les experts sont tous d'accord sur le mode de contre-financement de ces mesures : la réduction drastique de toutes les subventions. Mais aucun d'entre eux n'a encore formulé de proposition concrète sur ce point.

II est vrai qu'un régime fiscal plus transparent et débarrassé de ses innombrables dispositions particulières serait largement plus équitable. Car jusqu'ici, seuls ceux qui ont les moyens de se payer les services d'un conseiller fiscal réputé profitent de ces multiples possibilités : abattements, dégrèvements ou exonérations. En outre, une réforme de fond améliorerait considérablement l'attractivité du site fiscal allemand. II est donc décisif pour l'Allemagne qu'une réforme structurelle de la fiscalité soit engagée le plus rapidement possible.

\section{Quel avenir pour les réformes?}

Le gouvernement poursuivra-t-il sa politique de réformes ou en reviendra-t-il à cette « politique de la main tranquille » qu'il avait pratiquée en 2001 et 2002 ? Tout dépend si le gouvernement fédéral et, plus généralement, le monde politique, réussit à convaincre l'opinion de la nécessité de poursuivre les réformes.

Dans l'ensemble, les citoyens sont de plus en plus nombreux à accepter le principe des réformes structurelles. Mais dès que celles-ci s'en prennent au portemonnaie des uns ou au statut des autres, l'ardeur réformiste fléchit. Autrement dit : les réformes sont souvent adoptées contre une majorité de l'opinion. Ainsi, alors que près des trois quarts des Allemands approuvaient l'anticipation de l'entrée en vigueur du dernier volet de la réforme fiscale, les mesures liées à son financement (réduction des subventions, recours à la dette publique) se sont heurtées à une large opposition. Plus de $60 \%$ des Allemands étaient contre la suppression de la prime d'accession à la propriété et la réduction de l'abattement pour frais de transport. Les réformes en matière d'assurance maladie ou de régime des retraites avaient été adoptées elles aussi malgré l'opposition de l'opinion. Un cinquième seulement des Allemands approuvait comme juste et raisonnable le prélèvement d'une taxe de cabinet médical ou la hausse de la participation financière des assurés. Plus de la moitié des citoyens était contre le gel du niveau des pensions de retraite, mais un quart seulement d'entre eux aurait préféré à la place une hausse des cotisations retraite.

Le retour de bâton ne s'est pas fait attendre : lors des élections régionales de Bavière en septembre 2003, le parti social-démocrate a enregistré le plus faible taux de suffrages de son histoire. Quant au ministre-président CSU de Bavière, Edmund Stoiber, il doit son éclatante victoire aussi au fait qu'il n'a présenté son programme de réformes qu'après l'échéance électorale. Dans l'ensemble de l'Allemagne, le SPD a atteint son niveau de confiance historiquement le plus bas. Début novembre 2003, quelque 100000 manifestants protestaient à Berlin contre les réformes sociales du gouvernement fédéral SPD/Verts. Et pourtant, dans leur majorité, les Allemands sont conscients de la nécessité d'entreprendre une réforme de fond de l'Etat social et de ses piliers que sont l'assurance chômage, l'assurance retraite, l'assurance maladie ou l'assurance dépendance, pour en assurer durablement l'avenir.

Dans ce contexte, les 15 échéances électorales que compte l'année 2004 prennent une importance considérable. En effet, si la coalition fédérale abandonne le cap des réformes, les partis au gouvernement à Berlin pourront certes reconquérir quelques électeurs ici ou là. Mais parallèlement, ils verront augmenter le nombre de ceux qui, ne distinguant plus d'orientations politiques clairement définies, se détourneront du SPD et des Verts. Si, à l'inverse, la coalition fédérale maintient le cap des réformes, elle risque de perdre encore des voix. Mais en même temps, elle pourra asseoir définitivement son rôle de moteur du renouveau. Elle gagnera en crédibilité et, à moyen terme, elle en
Améliorer l'attractivité du site Allemagne, un enjeu décisif

Une opinion favorable sur le principe, pas sur le détail

Un vote sanction aux élections de Bavière en septembre 2003

2004, une année à haut risque électoral 
récoltera les fruits électoraux. Le dilemme est comparable pour l'opposition. Elle non plus n'a pas d'alternative à la poursuite des réformes structurelles.

2004 : une année où les élections s'enchaînent

\begin{tabular}{|c|c|}
\hline 29 février & Elections régionales* dans la ville-Etat de Hambourg \\
\hline 23 mai & Election du président de la République fédérale par l'Assemblée fédérale ${ }^{* *}$ \\
\hline 13 juin & $\begin{array}{l}\text { Elections parlementaires européennes } \\
\text { Elections régionales") en Thuringe } \\
\text { Elections communales dans les Länder : } \\
\text { - Bade-Wurtemberg } \\
\text { - Mecklembourg } \\
\text { - Rhénanie-Palatinat } \\
\text { - Sarre } \\
\text { - Saxe } \\
\text { - Saxe-Anhalt }\end{array}$ \\
\hline 27 juin & Elections communales dans le Land de Thuringe \\
\hline 5 septembre & Elections régionales") en Sarre \\
\hline 19 septembre & $\begin{array}{l}\text { Elections régionales") dans les Länder: } \\
\text { - Brandebourg } \\
\text { - Saxe }\end{array}$ \\
\hline 26 septembre & Elections communales dans le Land de Rhénanie du Nord-Westphalie \\
\hline
\end{tabular}

*) élections parlementaires débouchant sur le choix du nouveau gouvernement du Land ; **) elle se compose des membres du Bundestag et d'un nombre égal de députés des parlements des Länder.

MAIS LE GOUVERNEMENT ET L'OPPOSITION NE POURRONT CONQUÉRIR l'adhésion des citoyens aux réformes que s'ils les éclairent sans concession sur l'état réel du pays et les informent sur les objectifs et perspectives sur le long terme d'une politique de réformes convaincante. Cela n'a jamais été fait. Quand le monde politique se déterminera enfin à oser cette pédagogie des réformes, alors les chances seront grandes de voir l'Allemagne rompre définitivement avec l'immobilisme.

(Traduction : IB)

\section{L'Institut IWG, Bonn :}

Cet institut de recherche privé a été créé en 1977 par Kurt H. Biedenkopf et Meinhard Miegel. Il est spécialisé dans l'analyse des problèmes économiques et sociaux se posant sur le moyen et le long terme. Ses travaux

débouchent sur des recommandations pratiques.

Domaines de recherche :

- évolution de l'économie et de la sociéte allemande et européenne,

- cultures économiques et sociales,

- tendances démographiques quantitatives et qualitatives, - phénomènes migratoires et questions liées à l'immigration

- marché du travail,

- systèmes de protection sociale,

- finances publiques. www.iwg-bonn.de

\section{Indications bibliographiques :}

Miegel M., WAHL S., Solidarische Grundsicherung, Private Vorsorge - Der Weg aus der Rentenkrise, Schriften des IWG BONN, Olzog Verlag, Munich, 1999

Miegel M., Die deformierte Gesellschaft. Wie die Deutschen ihre Wirklichkeit verdrängen, Propyläen Verlag, Munich, 2002

OtTnAD A. et Schulte M., Die Pflegeversicherung : Ein Pflegefall. Wege zu einer solidarischen Absicherung des Pflegerisikos, Olzog Verlag, Munich, 2003

WAHL S., SCHULTE M., Arbeitsmarktreform - Überblick über aktuelle Reformkonzepte, ainsi que : Rentenreform - Überblick über aktuelle Reformkonzepte; Gesundheitsreform Überblick über aktuelle Reformkonzepte ; Pflegeversicherungsreform - Überblick über aktuelle Reformkonzepte et Steuerreform - Überblick über aktuelle Reformkonzepte, Gutachten im Auftrag des BürgerKonvent e.V., Bonn 2003

Voir aussi les numéros 51/01, 54/01, 59/02, 62/03 et 63/03 de Regards sur l'économie allemande (www.cirac.u-cergy.fr)

Sites Internet :

www.sachverstaendigenrat.org www.die-gesundheitsreform.de www.bmgs.bund.de

www.soziale-sicherungssysteme.de

www.bundesregierung.de

www.cdu.de/politik-a-z/herzogkommission/inhalt.htm

www.bundesfinanzministerium.de

www.cdu.de/politik-a-z/finanzen/inhalt.htm

www.fdp-bundesverband.de/kampagnen/steuern/steuerlinks.php

www.csu.de/home/uploadedfiles/Dokumente/040107_Konzept_21_Langfassung.pdf 